\title{
Cancer biology deciphered by single-cell transcriptomic sequencing
}

\author{
Yanmeng Li ${ }^{1}$ (D), Jianshi $\operatorname{Jin}^{2}$ (D), Fan Bai ${ }^{1,3 \bowtie}$ (I) \\ ${ }^{1}$ Biomedical Pioneering Innovation Center (BIOPIC), School of Life Sciences, Peking University, Beijing 100871, China \\ ${ }^{2}$ RIKEN Center for Biosystems Dynamics Research (BDR), 6-2-3, Furuedai, Suita, Osaka, Japan \\ ${ }^{3}$ Beijing Advanced Innovation Center for Genomics (ICG), Peking University, Beijing 100871, China \\ $\triangle$ Correspondence: fbai@pku.edu.cn (F. Bai)
}

Received July 5, 2021 Accepted July 21, 2021

\begin{abstract}
Tumors are complex ecosystems in which heterogeneous cancer cells interact with their microenvironment composed of diverse immune, endothelial, and stromal cells. Cancer biology had been studied using bulk genomic and gene expression profiling, which however mask the cellular diversity and average the variability among individual molecular programs. Recent advances in single-cell transcriptomic sequencing have enabled a detailed dissection of tumor ecosystems and promoted our understanding of tumorigenesis at single-cell resolution. In the present review, we discuss the main topics of recent cancer studies that have implemented singlecell RNA sequencing (scRNA-seq). To study cancer cells, scRNA-seq has provided novel insights into the cancer stem-cell model, treatment resistance, and cancer metastasis. To study the tumor microenvironment, scRNA-seq has portrayed the diverse cell types and complex cellular states of both immune and non-immune cells interacting with cancer cells, with the promise to discover novel targets for future immunotherapy.
\end{abstract}

KEYWORDS single-cell transcriptomic sequencing, tumor microenvironment, cancer

\section{INTRODUCTION}

Cancer is a genetic disease that continues to seriously threaten human health. Its evolutionary progression comprises the acquisition and accumulation of oncogenic somatic mutations and non-genetic alterations, which may lead to unrestrained proliferation, invasiveness, and treatment resistance of tumor cells (Weinberg, 2002; Pantel and
Brakenhoff, 2004; Merlo et al., 2006; Weis and Cheresh, 2011; Wan et al., 2013; Lambert et al., 2017; Maley et al., 2017). A comprehensive understanding of the molecular mechanisms underlying carcinogenesis is critical for diagnosis, drug development, clinical trial design and therapeutic strategy selection. In the past decade, next-generation sequencing (NGS) has advanced our understanding of cancer biology in many fields such as cancer genomics and transcriptomics (Cancer Genome Atlas Research, 2012a, b, 2014, 2016; Network, 2012a, b; Garraway and Lander, 2013; George et al., 2015; Wang et al., 2016; Nakagawa and Fujita, 2018), tumor evolution (Fearon and Vogelstein, 1990; Gerlinger et al., 2012; Sottoriva et al., 2015; Williams et al., 2016), intra-tumor heterogeneity (Gerlinger et al., 2012; Burrell et al., 2013; McGranahan and Swanton, 2017; Turajlic et al., 2019), and treatment resistance (Murugaesu et al., 2015; Swanton and Govindan, 2016; Chen and Mellman, 2017; Turajlic and Swanton, 2017; Berger and Mardis, 2018; Binnewies et al., 2018). Although great achievements have been made, most conventional analyses and datasets are from bulk samples, which average the molecular features of cells in a tumor sample.

Tumors are increasingly viewed as complex ecosystems, in which heterogeneous cancer cells interact with their microenvironment composed of, but not limited to, diverse immune, endothelial, and stromal cells (Chen and Mellman, 2017; Binnewies et al., 2018; Valkenburg et al., 2018). Emerging evidence show that not only the cancer cells themselves but also the cells in the microenvironment shape the biological and clinical behavior of a tumor. This insight suggests that bulk profiling of tumors may mask the cellular diversity and is hard to study the molecular mechanisms of different cell types in a tumor which may contribute to carcinogenesis in distinct ways. Therefore, to handle the cell heterogeneity in tumors, the recently developed single-cell 
sequencing technologies (Tang et al., 2009; Navin et al., 2011; Baslan et al., 2012; Ramskold et al., 2012; Picelli et al., 2013; Klein et al., 2015; Hashimshony et al., 2016; Baslan and Hicks, 2017; Zheng et al., 2017b) have been utilized in many cancer studies to achieve an understanding of tumors at the resolution of individual cells. In the present review, we focus mainly on recent cancer studies that have implemented single-cell RNA sequencing (scRNA-seq) and we summarize their relevant themes and results. Meanwhile, we discuss the therapeutic implications of these new knowledge and discoveries.

\section{INTRICATE TUMOR ECOSYSTEMS}

In a tumor, the diverse malignant cells, nonmalignant cells, and non-cellular factors interact synergistically to form a complicated "tumor ecosystem" that ultimately underlies many critical facets of tumor biology (Fig. 1A) (Suva and Tirosh, 2019). The tumor ecosystem mainly contains two parts: the tumor cells (i.e., malignant cells) and the tumor microenvironment (TME) (i.e., nonmalignant cells and noncellular factors).

Regarding malignant cells, intra-tumor heterogeneity (ITH) is an inherent property influencing tumor growth, metastasis, recurrence, and response to therapies. Cell-tocell expression ITH demonstrated by scRNA-seq can be interpreted at three levels (Tirosh and Suva, 2019): (1) genetic heterogeneity, in which subpopulations of tumor cells harbor subclonal mutations that are selected for during cancer evolution; (2) non-genetic heterogeneity, consisting of diverse epigenetic and differentiation programs; and (3) environmental and spatial factors related to the supply of nutrients, oxygen, and inter-cell interactions. Dissection of tumor architecture, especially on the expression level, can vastly enhance our knowledge of the molecular mechanisms related to the development and treatment resistance of cancer.

On the other hand, the TME plays a crucial role in shaping the tumor behavior. The TME comprises various immune cells ( $T$ cells, B cells, myeloid cells), stromal cells (fibroblasts, osteoblasts), other resident host cells (endothelial cells, adipocytes), and factors secreted by these cells, and non-cellular constituents of the extracellular matrix (ECM) (Maman and Witz, 2018). To describe the effect of the TME on tumor phenotype, models including the "seed-and-soil" hypothesis and the ecosystem networks are proposed (Amend et al., 2016; de Groot et al., 2017). These models highlight the effect of the TME components on regulating and remodeling tumor behavior. Multiple mechanisms are illustrated: (1) ligand-receptor crosstalk, Cells in the TME secrete multiple ligands that can be sensed by cancer cells or other cells, leading to the alteration of specific signaling pathways; (2) cell-cell interaction, such as the interaction between cytotoxic effector $\mathrm{CD}^{+} \mathrm{T}$ cells and tumor cells; and (3) cancer cells experiencing stress from the TME, such as hypoxia, starvation, and DNA damage (Maley et al., 2017).
Furthermore, the TME contributes to the spatiotemporal inter- and intra-tumor heterogeneity. For example, there are three subclasses of tumor immune microenvironment that dictates tumor response to therapy: infiltrated-excluded, infiltrated-inflamed, and tertiary lymphoid structure (Binnewies et al., 2018). More importantly, different cell types and their diverse states in the TME can implement either anti-tumor or pro-tumor functions during cancer progression (Klein-Goldberg et al., 2014); thus, the entire tumor ecosystem should be considered when assessing the feasibility of a therapeutic approach.

Due to the complexity of the tumor ecosystem, analysis of a tumor at single-cell resolution, e.g., using scRNA-seq, is required. Benefiting from the high-throughput and unbiased gene expression analysis of scRNA-seq, not only cell types such as T cell, B cell, fibroblast, and tumor cell clusters, but also cell states within each cell-type (i.e., subclusters) can be identified in the tumor ecosystem (Fig. 1B) (Suva and Tirosh, 2019), by using the expression levels of canonical cell markers and/or genetic alterations such as large-scale copy number variations (Filbin et al., 2018). It is important to distinguish the cell states of the same cell type, because different gene expression of these states are related to cell cycle stage, different metabolic processes, and other dynamic programs. Moreover, rare or even new cell types with specific functional properties, such as stemness, inflammation, self-renewal, invasiveness, and drug resistance, can also be captured and characterized. Thus, precise identification of diverse cell types and states has both significant biological and clinical implications.

\section{CANCER CELL BIOLOGY ILLUMINATED BY SCRNA- SEQ}

In this section, we discuss research themes from recent scRNA-seq-based cancer studies that have linked heterogeneous expression programs of tumor subpopulations to clinical phenotypes.

\section{Tracing the cell type of tumor origin}

Investigation of the initiation of tumor, e.g., identifying the types and states of cells from which malignancy originates, is an essential part of cancer research, because the cell type of tumor origin serves as a credible criterium for cancer classification. To identify the origin of cancer cells, prior knowledge of the cellular compositions and hierarchies of normal tissue is required (Vermeulen and Snippert, 2014). Singlecell RNA analysis enables deciphering of the constitution of normal tissues and subsequent lineage tracing. For example, in a recent scRNA-seq analysis, researchers found that tumor cells from distinct molecular subgroups of childhood cerebellar tumor mirror the transcriptional programs of different, temporally restricted cerebellar lineage cells (Vladoiu et al., 2019). They found that the Sonic Hedgehog medulloblastoma subgroup transcriptionally resembled the granule 
A

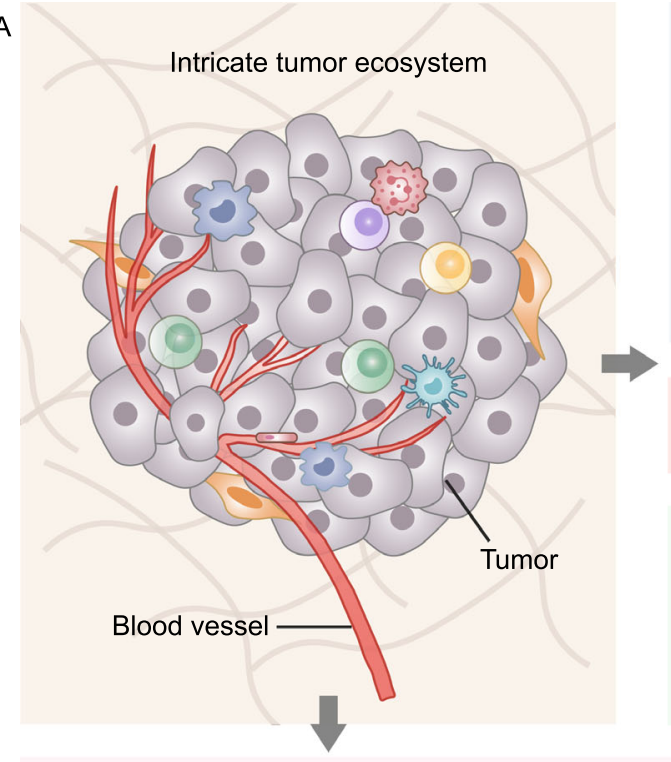

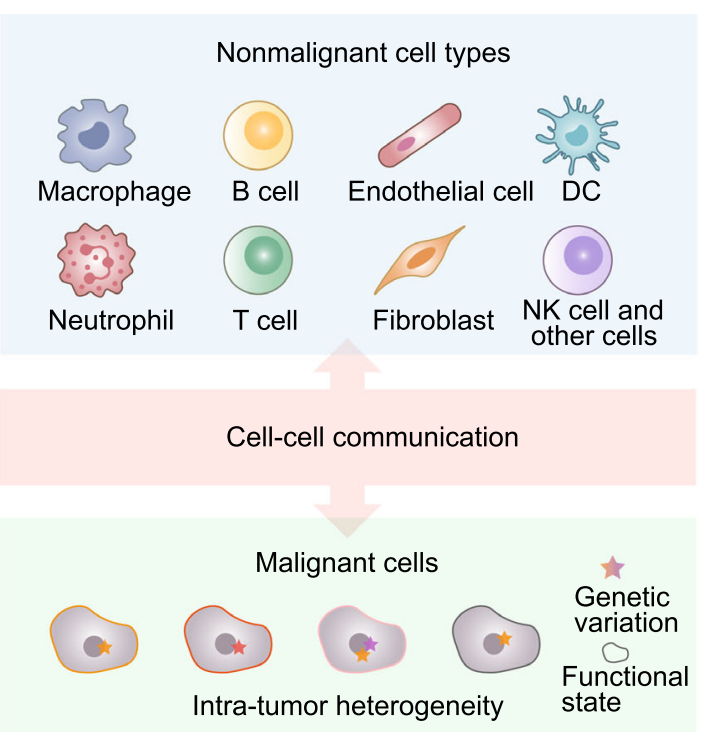

Diverse tumor phenotypes

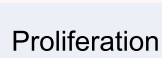

Migration

Invasion

Immunotherapy response

The underlying mechanisms

B

Drug resistance

Single-cell expression data

- CNV inference - Bioinformatic clustering
- Functional programs

Cell states within each cell-type cluster

Cell types (based on canonical markers)

Figure 1. Intricate tumor ecosystem and the workflow for scRNA-seq analysis of tumors. (A) Tumors are complex ecosystems (left) composed of diverse malignant and nonmalignant cells (right). Nonmalignant cells include immune, endothelial, and stromal cells. Intra-tumor heterogeneity is an inherent feature of malignant cells mainly contributed by genetic heterogeneity and functional expression programs. These complicated cancer ecosystems underlie many critical facets of tumor biology. (B) Tumors are digested and sorted into single live cells and then profiled by scRNA-seq. Copy number variation (CNV) inference and canonical makers are used to annotate cell types. Cell states within each cell-type cluster are identified by bioinformatics clustering and functional programs. Advances in scRNA-seq have enabled a detailed dissection of tumor entities and enhanced our understanding of the underlying mechanisms at the resolution of individual cells.

cell hierarchy, group 3 medulloblastoma mirrors Nestin $(+)$ stem cells, group 4 medulloblastoma resembles unipolar brush cells, and PFA/PFB ependymoma and cerebellar pilocytic astrocytoma mirror prenatal gliogenic progenitor cells. Their results support the view that cerebellar tumors are a disorder from early brain development, revealing the origin of cerebellar cancer cells. In a similar study of renal cancer, investigators profiled 72,501 single-cell transcriptomes of human renal tumors and normal tissues from fetal, pediatric, and adult kidneys (Young et al., 2018). They found that cells from childhood Wilms tumors were matched with specific fetal cell types, and a canonical cancer transcriptome that matched a little-known subtype of proximal convoluted tubular cells in adult renal cell carcinoma was 
identified (Young et al., 2018). In another study, a human liver cell atlas was constructed (Aizarani et al., 2019), which has been used as a reference for the comparison of liver cancer cells. Through differential gene expression analysis, it was found that liver cancer cells lose the expression of cytochrome P450 genes, such as CYP2E1 and CYP2C8, and the periportally zonated gene CPS1, and show increased expression of $A K R 1 B 10$, which is related to hepatocellular carcinogenesis. More recently, Guo et al. characterized the prostate epithelial cell lineage hierarchy from mouse prostates using scRNA-seq (Guo et al., 2020). They identified a unique luminal cell type (termed type $C$ luminal cell (Luminal-C)) marked by Tacstd2, Ck4 and Psca expression. Functional experiments showed that Luminal-C cells which located at the distal prostate invagination tips had great capacity for organoid formation in vitro and prostate epithelial duct regeneration in vivo. They further identified the existence of Luminal-C cells in human prostate and suggested these cells can serve as the cellular origin of prostate cancer.

In summary, scRNA-seq is a powerful tool to delineate the cell types of tumor origin, and to identify their gene expression signatures and biomarkers.

\section{Developmental hierarchy and the cancer stem-cell} model

Decades of studies provide evidence that tumor differentiation is controlled by a rare subset of cancer cells, termed "cancer stem cells" (CSCs) which possess many properties of stem cells (Dean et al., 2005; Clevers, 2011; Kreso and Dick, 2014). Similar to stem cells in normal tissues, CSCs are able to renew themselves and contribute to establish cellular hierarchies and heterogeneity of a tumor. Moreover, CSCs are thought to play important roles in therapy resistance and cancer relapse. Despite the cancer stem-cell model has been developed for decades (Hermann et al., 2007; Jan et al., 2012; Yamamoto et al., 2013; Lawson et al., 2015), the cellular states and putative markers of CSCs are far from clear in tumor samples.

scRNA-seq provides a unique avenue to uncover the cellular hierarchy of cancer cells and to identify CSCs. Using scRNA-seq, researchers found that primary glioblastoma cells display a spectrum of stemness and differentiation potential, revealing a putative CSC expression program (Patel et al., 2014). They also demonstrated that several transcription factors, such as POU3F2, NFIA, and NFIB, are important to regulate the stem-like phenotype. In other studies, researchers performed scRNA-seq on isocitrate dehydrogenase (IDH)-mutant oligodendroglioma (Tirosh et al., 2016b) and astrocytoma (Venteicher et al., 2017), revealing similar developmental hierarchies and lineages of glial differentiation of tumor cells. These two studies supported the cancer stem-cell model, in which the majority of cancer cells are well-differentiated and remain as oligodendrocyte-like or astrocyte-like cell lineages; however, a subset of undifferentiated cells possess properties of stem/ progenitor cells. Of note, stem-like glioma cells with signatures of proliferation are enriched as the tumor grade increases, indicating that this minority group of cancer cells makes a large contribution to the growth and progression of IDH-mutant glioma. Furthermore, Filbin et al. demonstrated a similar cellular hierarchy and a subset of stem-like cells that resemble oligodendrocyte precursor cells (OPC-like) in primary H3K27M-glioma (Filbin et al., 2018), which constitute a higher fraction and exhibit greater tumor-propagating potential than their more differentiated counterparts. To investigate whether the cell state diversity within a tumor is determined by genetic factors, copy number variation (CNV) sub-clones inferred from scRNA-seq along with expression profiles were used to study the relationship between genetic subclones and intra-tumor cellular state diversity. For instance, in IDH1 or IDH2 mutant human oligodendrogliomas (Tirosh et al., 2016b), distinct CNV sub-clones displayed similar cellular hierarchies, suggesting that the cell states were primarily dictated by developmental programs. Consistent with this conclusion, an integrative and multiomics study of glioblastoma (Neftel et al., 2019) revealed that the cellular hierarchy covered four cell states and these states were not strictly determined by CNV patterns. In glioblastoma, the CSC model was validated using functional experiments, which further explained why different cellular states remarkably converge to the same distribution of states in both patient-derived xenograft (PDX) models and patients (Neftel et al., 2019). In summary, scRNA-seq studies on gliomas support the cancer stem-cell model, implying that CSCs can act as potential targets for a better control of tumor differentiation and malignant progression.

In addition to gliomas, a subgroup of tumor cells displaying stem-like features are also found in other solid cancer types, such as prostate cancer (Horning et al., 2018), breast cancer (Savage et al., 2017), and hepatocellular carcinoma (Zheng et al., 2018). In prostate cancer, the advanced growth of the CSCs is related to attenuated androgen response and enhanced expression of cell cyclerelated genes, which foster the plasticity of prostate cancer cells regarding their androgen dependence (Horning et al., 2018). In breast cancer, mesenchymal/stem-like tumor cells are present in EGFR inhibition responders (Savage et al., 2017). EGFR expression-high subpopulations show enhanced stem-like features, illustrating an EGFR-dependent hierarchy and enhancing the understanding of patient stratification for therapeutic intervention. As shown in a scRNA-seq analysis of hepatocellular carcinoma (Zheng et al., 2018), CSCs are phenotypically, functionally, and transcriptomically heterogeneous. Moreover, studies on the developmental hierarchies and the molecular signatures of stem cells of leukemia are instrumental for designing the molecularly targeted cancer therapies. In acute myeloid leukemia (AML), Velten et al. identified the gene expression programs of leukemic stem cells (LSCs) by combining 
scRNA-seq with lineage tracing using both nuclear and mitochondrial somatic variants (Velten et al., 2021). They highlighted that FOS and CD96 may serve as important markers for LSCs. Meanwhile, Galen et al. provided an atlas of AML cell states and revealed the hierarchies related to disease progression (van Galen et al., 2019). The primitive AML cells were characterized by dysregulated transcriptional programs with co-expression of stemness and myeloid priming genes. Differentiated AML cells expressed diverse immunomodulatory genes and suppressed $T$ cell activity in vitro. In chronic myeloid leukemia (CML), researchers identified distinct molecular signatures of CSCs and revealed their heterogeneity (Giustacchini et al., 2017). Notably, they uncovered a subgroup of CML stem cells with a distinct molecular signature that selectively persisted during prolonged TKI therapy. These therapy-resistant stem cells were characterized by marked quiescence-associated gene expression and showed dysregulation of specific genes and pathways (TGF- $\beta$, TNF- $\alpha$, JAK-STAT, CTNNB1 and NFKB1A). These findings provide great insights into the cellular and molecular mechanisms of therapy resistance in CML.

Overall, scRNA-seq studies of tumors provide insight into the cellular states of CSCs and the molecular mechanisms of cellular hierarchy. However, the programs and biomarkers of CSCs should be further identified and verified by functional studies, which may contribute to the development of therapies targeting CSCs in tumor.

\section{Treatment resistance}

A central problem in cancer therapy is that the majority of patients are unresponsive or develop acquired resistance. This issue can be experimentally interpreted using scRNAseq to discover subsets of cancer cells with drug-susceptible or drug-resistant phenotypes, and to unravel their underlying programs. For example, a single-cell study in melanoma showed that two distinct transcriptional cell states, characterized by high levels of MITF expression and low MITF expression with elevated levels of AXL kinase, coexist in the same tumor (Tirosh et al., 2016a). The high-AXL program has been linked to intrinsic resistance to RAF and MEK inhibition, suggesting a subpopulation of high-AXL cells are involved in drug resistance and appear to increase upon treatment and recurrence. Rambow et al. reported that distinct drug-tolerant transcriptional states of single malignant cells were identified in the minimal residual disease of BRAF-mutant melanoma (Rambow et al., 2018). In this study, a neural crest stem cell transcriptional program, largely driven by the nuclear receptor RXRG, was found to mediate the development of resistance. In addition to resistance of targeted therapy, chemotherapy/radiotherapy resistance has also been studied by scRNA-seq. For instance, in nasopharyngeal carcinoma, a subset of malignant cells with an epithelial-immune dual feature was identified and found to be associated with poor prognosis (Jin et al., 2020). This clinical association is partly due to a higher expression level of genes (e.g., STAT1, ISG15 and IFIT1) that are potentially related to DNA damage resistance and chemotherapy/radiotherapy resistance. Furthermore, similar studies regarding resistance to cancer immunotherapy were also performed. For example, Paulson et al. reported that two patients with metastatic Merkel cell carcinoma responded well at first, but finally developed late relapse, to the treatment with immune-checkpoint inhibitors and autologous Merkel cell polyomavirus-specific $\mathrm{CD}^{+} \mathrm{T}$ cells (Paulson et al., 2018). Using scRNA-seq, this study also identified dynamic transcriptional suppression of HLA-specific genes that can present the targeted viral epitope under intense CD8-mediated immunologic pressure, suggesting that the transcriptional suppression of Class I loci may underlie resistance to immunotherapies. Another study on melanoma tumors treated with immune checkpoint inhibitors (ICls) provided a high-resolution landscape of the ICl-resistant cell state, which is associated with T cell exclusion and immune evasion (Jerby-Arnon et al., 2018). Recently, Miao et al. performed analysis on a model of squamous cell carcinoma treated with adoptive cytotoxic T cell transfer (ACT)-based immunotherapy, showing that TGF- $\beta$-responsive tumor-initiating stem cells are superior at resisting ACT (Miao et al., 2019). These cells selectively acquire CD80 (a surface ligand previously identified on immune cells) and CTLA4 to directly dampen cytotoxic $T$ cell activity. In addition to the above-mentioned studies that demonstrated the mechanisms underlying adaptive and acquired resistance, other studies have identified subsets of circulating tumor cells (CTCs) with potential programs of drug resistance by comparing tumors prior to and following treatment (Patel et al., 2014; Miyamoto et al., 2015; Jordan et al., 2016).

\section{Metastasis}

Cancer metastasis, the dissemination of tumor cells from the primary site and colonization in distant organs, causes the majority of cancer-related deaths. A series of scientific problems related to cancer metastasis, such as the variable dissemination abilities of tumor subpopulations and the transcriptional alterations involved in metastasis, as well as the different responses of the primary tumor and metastases to the same drug, can be explored using scRNA-seq. A recent study compared primary renal cell carcinoma and its lung metastases and found considerable variability in the activation of certain drug-target pathways (Kim et al., 2016). Just et al. compared primary $\mathrm{ER}^{+}$breast cancer cells and their luminal UCD65 brain or luminobasal UCD46 liver metastases, and defined the unique molecular determinants of organ-specific metastasis (Just et al., 2018). Similarly, a study compared primary head and neck squamous cell carcinomas (HNSCCs) with their matched lymph node metastases, and showed that a partial epithelial-to-mesenchymal transition ( $p$-EMT) program was spatially localized to the leading edge of each primary tumor, which was 
confirmed to be associated with metastases (Puram et al., 2017). These results suggested that the $p$-EMT signature may play a vital role in the initiation of metastatic dissemination but then disappear in the corresponding metastases. To understand the mechanism underlying metastasis, Chung et al. characterized the expression signatures and programs that promote metastatic progression in several rare subsets of primary triple-negative breast cancer using scRNA-seq, and identified strong EMT and stemness signatures, which may drive tumor progression and metastasis (Chung et al., 2017). This phenomenon was confirmed by another study, in which the potentially metastatic cells within migratory breast cancer cells exhibit overall signatures of EMT and stemness with variable expressions of marker genes (Pastushenko et al., 2018). Interestingly, a study on primary skin and mammary tumors identified different EMT stages in multiple tumor subpopulations, including intermediate hybrid states between completely epithelial and completely mesenchymal (Pastushenko et al., 2018; Chen et al., 2019). Functional experiments demonstrated that tumor cells with hybrid epithelial and mesenchymal phenotypes are more competent in reaching the circulation, colonizing the lungs, and forming metastatic sites. However, the molecular mechanisms underlying tumor metastatic dissemination and the detailed difference between primary tumors and metastases are still poorly understood, which requires further studies using scRNA-seq.

\section{TUMOR MICROENVIRONMENT PORTRAYED BY SCRNA-SEQ: ONE CELL AT A TIME}

It has been increasingly recognized that non-malignant cellular components of the TME, including diverse subsets of lymphocytes, myeloid cells, stromal cells, other resident host cells, and their secreted factors, have a significant impact on cancer progression and treatment outcomes (Chen and Mellman, 2017; Maman and Witz, 2018; Ren et al., 2018). Importantly, the TME can apply both pro- and anti-tumorigenic functions, which are contributed by various cell types and/or states as well as their dynamic changes. Therefore, it is important to characterize the various components of the TME and their dynamic changes, which may help to develop novel therapies. In this section, we review some recent progress in studying tumor-infiltrating lymphocytes, myeloid cells, and stromal cells using scRNA-seq.

\section{Tumor-infiltrating lymphocytes (TILs)}

Within tumor-infiltrating immune cells, tumor-infiltrating lymphocytes (TILs), mainly comprised of T cells, B cells, and NK cells, can display both cytotoxic functions to directly eradicate malignant cells and pro-tumorigenic activities to facilitate the progression of cancer.

Among these TILs, tumor-infiltrating T cells are the major determinants of tumor behavior and the major responder to cancer immunotherapies, such as T cell immune-checkpoint blockade therapies (Chen and Mellman, 2017; Binnewies et al., 2018). Recently, the heterogeneous tumor-infiltrating $T$ cells have been studied using scRNA-seq and their crucial roles have been understood from the following aspects.

First, multiple $\mathrm{T}$ cell subsets including rare/new cancerassociated subtypes have been systematically identified. Using scRNA-seq, the tumor-infiltrating $T$ cell landscapes were characterized in head and neck cancer (Cillo et al., 2020), hepatocellular carcinoma (Zhang et al., 2019), rhabdoid tumors (Leruste et al., 2019), colorectal cancer (Zhang et al., 2018), breast cancer (Azizi et al., 2018; Savas et al., 2018), non-small-cell lung cancer (Guo et al., 2018), nasopharyngeal carcinoma (Jin et al., 2020), and melanoma (Tirosh et al., 2016a). For instance, Zheng et al. performed full-length scRNA-seq in 5,063 single T cells obtained from six hepatocellular carcinoma patients and identified $11 \mathrm{~T}$ cell subsets with different molecular and functional features (Zheng et al., 2017a). A similar study conducted by the same group depicted the landscape of infiltrating $T$ cells in nonsmall cell lung cancer by analyzing 12,346 T cells from 14 patients, demonstrating 16 main T cell subsets including 7 $\mathrm{CD}^{+} \mathrm{T}$ cell subsets, 7 conventional CD4 ${ }^{+} \mathrm{T}$ cell subsets, and 2 regulatory $T$ cell (Tregs) subsets (Guo et al., 2018). In a recent study depicting the tumor ecosystem of early-stage lung adenocarcinomas that present as subsolid nodules (SSNs), researchers compared the relative percentage of various T cell subsets in SSNs to those in normal lung tissue (nLung) and those in advanced-stage LUAD with metastasis (mLUAD) (Xing et al., 2021).

In addition to quantifying these well-known $T$ cell subtypes, some rare functional clusters specific to the tumor context have been identified as well, because of the unprecedented resolution offered by scRNA-seq. For example, a $\mathrm{CD}^{+}$tissue-resident memory $\mathrm{T}$ cell subset, distinct from conventional Teffector memory cells, was found in breast cancer (Savas et al., 2018). In detail, high expression levels of immune checkpoints (such as TIM3, PD1, CTLA4, TIGIT, and LAG3) and effector proteins (including granzyme $B$ and perforin) were observed in these cells. Moreover, the identified gene signature of this $T$ cell subset is significantly associated with improved survival in triple-negative breast cancer, providing improved prognostic information. In another example, a newly defined $\mathrm{T}$ cell subset in colorectal cancer, a group of $\mathrm{CXCL} 13^{+} \mathrm{BHLHE} 40^{+}$ TH1-like cells, was found to be enriched in microsatelliteunstable tumors (Zhang et al., 2018). These cells express a high level of IGFLR1, which shows a co-stimulatory function to enhance the production of IFN- $\gamma$, and also exhibit high clonal expansion and proliferation. This finding may explain why patients with microsatellite-instable colorectal tumors respond well to immune checkpoint blockade, and may offer a new therapeutic target in immune therapy.

Secondly, the functional state transition and lineage connection of distinct tumor-infiltrating T cell subtypes have been deciphered: T cells can enter into different states with distinct functional behaviors. For instance, effector $\mathrm{T}$ cells 
can suppress tumor progression by lysing cancer cells, whereas dysfunctional or exhausted effector $\mathrm{T}$ cells with elevated expression of inhibitory receptors (e.g., PD-1, TIM3, CTLA-4, LAG3, and TIGIT) impair antitumor effector functions (Zhang and Zhang, 2019). Using scRNA-seq, dysfunctional states of tumor-infiltrating $T$ cells and their expression signatures and regulators have been characterized in several studies (Singer et al., 2016; Tirosh et al., 2016a; Zheng et al., 2017a; Guo et al., 2018; Zhang et al., 2018, 2019). Moreover, the dynamic relationships among different $\mathrm{T}$ cell states were also unraveled. For example, a model of continuous $\mathrm{T}$ cell activation was identified in breast cancer (Azizi et al., 2018), in addition to a model of dysfunction with an underlying gene module uncoupled from $T$ cell activation (Singer et al., 2016). Furthermore, scRNA-seq combined with single-cell TCR analysis has given further insight into the developmental connection among $\mathrm{T}$ cell states. Using both techniques, Zhang et al. demonstrated that the $\mathrm{CD}^{+} \mathrm{GZMK}^{+}$memory $\mathrm{T}$ cells have a lineage connection with $\mathrm{CXCL} 13^{+} \mathrm{TH} 1$-like cells in colorectal cancer, suggesting these two subsets have developmental relationship (Zhang et al., 2018). Similarly, exhausted T cells have developmental connections with $\mathrm{GZMK}^{+} \mathrm{T}$ cells, a subtype related to effector T cells, in both liver and colorectal cancers (Zheng et al., 2017a; Zhang et al., 2018, 2019). Two "preexhausted" $T$ cell subsets including $\mathrm{CD}^{+} \mathrm{GZMK}^{+}$and $\mathrm{CD}^{+}{ }^{+} \mathrm{ZNF} 683^{+} \mathrm{T}$ cells have been identified in non-small-cell lung cancer (Guo et al., 2018). In accordance with the continuous transition model of exhaustion, a recent study in human melanoma identified a gradient of T cell dysfunction, demonstrating a separation between bystander cytotoxic $\mathrm{T}$ cells and a transitional-to-dysfunctional lineage ( $\mathrm{Li}$ et al., 2019). This study also showed that, surprisingly, the exhausted T cells are highly proliferative and clonal and their related dysfunctional signature was shown to be linked to the tumor reactivity of the T cell pool. These results facilitate the understanding of the regulatory mechanisms underlying $\mathrm{T}$ cell state transition and the identification of new immunotherapy targets.

Thirdly, the features of treatment-response expression of T cells and their changes have been depicted, which provide new insights into the $\mathrm{T}$ cell response to treatment. For example, by analyzing $\mathrm{CD}^{+} \mathrm{T}^{-}$cell pools prior to and following combined $\mathrm{Tim}^{-} 3^{+}$and PD-1-blockade treatment, an induced shift from naive-like to memory precursor-like and effector-like PD-1-CD8 ${ }^{+}$T cell subsets was identified (Kurtulus et al., 2019). Moreover, the transcriptional regulator Tcf7/Tcf1 was found to be necessary for maintaining memory-precursor $\mathrm{T}$ cells and the efficacy of immunotherapies. Another study analyzed $\mathrm{T}$ cells from patients with basal or squamous cell carcinoma prior to and following anti-PD-1 therapy and showed that the response to therapy depends on the newly recruited $T$ cells rather than the reinvigoration of pre-existing T cells (Yost et al., 2019). Recently, Bi et al. characterized the single-cell transcriptomes of cancer and immune cells from advanced renal cell carcinoma patients before or after immune checkpoint blockade treatment $(\mathrm{Bi}$ et al., 2021). They found that $C D 8^{+} \mathrm{T}$ cell phenotypes were reprogrammed to express higher levels of co-inhibitory receptors (PDCD1, TIGIT, and HAVCR2) and effector molecules (GZMB, PRF1, and IFNG) in responders.

In addition to T cells, among the TILs, tumor-infiltrating $B$ cells and NK cells are also active regulators of tumor progression and treatment response. Increasing evidence has shown that tumor-infiltrating NK cells can function in both anti-tumor cytotoxic and ineffective states (Sceneay et al., 2012; Brodbeck et al., 2014), and some tumor-infiltrating B cell populations exhibit pro-malignancy functions (Affara et al., 2014; Pylayeva-Gupta et al., 2016). Recently, the diverse subtypes and gene signatures of tumor-infiltrating $B$ cells and NK cells have been characterized in several cancer types using scRNA-seq (Tirosh et al., 2016a; Chung et al., 2017; Lavin et al., 2017; Zhang et al., 2019; Cillo et al., 2020). For instance, in head and neck squamous cell carcinoma (HNSCC), 11 B cell subsets were identified, and they presented distinct distributions between HPV- and HPV+ HNSCCs (Cillo et al., 2020): HPV+ HNSCCs contain a greater number of $\mathrm{B}$ cells which are more in non-germinal center states than HPV- HNSCC. Moreover, two other scRNA-seq studies highlighted the important role of tumorinfiltrating $B$ cells in mediating tumor response to therapies. In detail, Hollern et al. created novel mammary tumor models and revealed that $T$ cell activation mediated by $B$ cells and antibodies generation are essential in immunotherapy response (Hollern et al., 2019). Another study identified an emerging $\mathrm{ICOSL}^{+} \mathrm{B}$ cell subset after neoadjuvant conventional chemotherapy (Lu et al., 2020), and showed that the opposite functions of B cells during chemotherapy were shaped by complement signals and determined by CD55 expression, providing important implications for future antitumor therapies.

\section{Tumor-infiltrating myeloid cells (TIMs)}

In addition to TILs, tumor-infiltrating myeloid cells (TIMs) also play an important role in shaping tumor behavior. The diverse TIMs, mainly tumor-associated macrophages (TAMs), dendritic cells (DCs), and neutrophils, possess different and dynamic anti- or pro-tumorigenic functions (Binnewies et al., 2018; Maman and Witz, 2018), directly interacting with tumor cells and can modify the activities of other cells (Salmon et al., 2016). Recently, extensive heterogeneity among TIMs has been revealed by delineating the landscape of the major cell types and states of TIMs in head and neck squamous cell carcinoma (Cillo et al., 2020), hepatocellular carcinoma (Zhang et al., 2019), melanoma ( $\mathrm{Li}$ et al., 2019), rhabdoid tumors (Leruste et al., 2019), nonsmall cell lung cancer (Song et al., 2019), and breast cancer (Azizi et al., 2018) using scRNA-seq. For example, in human melanoma, multiple subsets of TIMs containing macrophages, monocytes, DCs, plasmacytoid DCs, and a small population of osteoclast-like cells were identified ( $\mathrm{Li}$ et al., 
2019). Similarly, in breast cancer, subsets of TIMs are also complicated and were delineated in four branches in the cell activation diffusion map (Azizi et al., 2018): one branch almost totally covers TAMs from three subsets; two branches capture a gradual trace from blood monocytes to intra-tumoral monocytes; and one branch distinguish a plasmacytoid DC subset from other monocyte subsets. Different from the above cancers, the composition of TIMs in rhabdoid tumors (genomically simple pediatric cancer) is simple, which was identified as DCs, monocytes, and TAMs (Leruste et al., 2019).

Among TIMs, TAMs show great functional and molecular plasticity, so that TAMs are a hot research topic in cancer research. It has been known from conventional studies that distinct cellular states of TAM have positive or negative roles in cancer. For example, M1-macrophages are considered to possess anti-tumor functions by phagocytosing and producing Th1-promoting cytokines, such as IL-12, IL-23, and TNF- $\alpha$, while M2-macrophages are thought to have pro-tumor functions by regulating angiogenesis, suppressing immune surveillance, promoting inflammation, and stimulating cancer cell motility by secreting growth factors and chemokines (IL-10, TGF- $\beta$, PD-L1, and ARG-1) (Engblom et al., 2016). Recently, more extensive heterogeneity among TAMs was revealed using scRNA-seq. For instance, TAM subsets with distinct molecular and functional features were comprehensively identified in rhabdoid tumors (Leruste et al., 2019); there are a subset of macrophages expressing APOE, FOLR2, RNASE1, and the genes defining macrophage activation signature, a subset of macrophages expressing $M M P 9$, cathepsin $\mathrm{K}$, and proteases relating to tissue remodeling, and a subset of macrophages expressing HMOX, an enzyme with anti-inflammatory properties. Of note, the genes related to angiogenesis, immunosuppressive functions, and M2-like signature are significantly enriched in the $\mathrm{APOE}^{+}$macrophages. Furthermore, scRNA-seq identifies a controversial issue whether TAMs conform to the polarization model in different cancers. In lung cancer, macrophages show rheostatic phenotypes and become M2polarized in tumors, suggesting their detrimental activities in the TME (Lambrechts et al., 2018). However, TAMs in breast cancer (Azizi et al., 2018), liver cancer (Zhang et al., 2019), and gliomas (Muller et al., 2017) frequently co-express M1 and M2 gene signatures in individual cells, arguing a more complicated pattern than the classical M1/M2 model. These studies suggested that further analysis of the differential trajectories and functional identification of TAMs should be performed. Similar to macrophages, DCs have also been demonstrated to exhibit adverse functions in the TME (Song et al., 2019; Zhang et al., 2019; Cillo et al., 2020). For instance, a subset of tumor-infiltrating LAMP3 $^{+}$DCs was identified in liver cancer and was shown to express various immune-relevant ligands with the potential ability to regulate multiple subtypes of lymphocytes (Zhang et al., 2019). Different from the dual character of macrophages and DCs, neutrophils have been shown that certain types always exert pro-tumor functions (Maman and Witz, 2018). For instance, using scRNA-seq, neutrophils were found to improve cell cycle progression of circulating tumor cells, which increased the probability of cancer metastasis (Szczerba et al., 2019).

\section{Stromal cells}

As a vital part of the TME, non-parenchymal cells of the stroma in the tumor niche, mainly fibroblasts and osteoblasts, exert notable effects on many hallmarks of cancer. Tumor associated stromal cells regulate the behaviors of tumor cells in several ways, such as remodeling and degrading the extracellular matrix (ECM) by secreting collagen and proteolytic enzymes; promoting EMT in tumor cells; recruiting or regulating immune cell infiltration; and releasing cytokines, chemokines, and growth factors (Orimo and Weinberg, 2006; Maman and Witz, 2018; Valkenburg et al., 2018). Although the active role of stromal cells within tumors is already appreciated, the detailed repertoire in different cancer types remained elusive until recently. For example, a systematic census of stromal cells in lung cancer was provided using scRNA-seq (Lambrechts et al., 2018), in which a total of 53 stromal cell subtypes including novel subpopulations were characterized. Similarly, a cellular taxonomy of bone marrow stroma in homeostasis and leukemia was characterized using scRNA-seq (Baryawno et al., 2019), in which 17 stromal subsets with distinct hematopoietic regulatory genes covering new mesenchymal, pericyte, fibroblast, and endothelial subsets were identified. This study also showed that impaired mesenchymal osteogenic differentiation and reduced regulatory molecules are necessary for normal hematopoiesis to occur in leukemia. These findings deepen our understanding of stromal cells perturbed by malignancy.

The dominant constituents of the tumor stroma cells are cancer-associated fibroblasts (CAFs), which always harbor pro-malignancy properties and are often referred to as myofibroblasts due to their altered shape and expression of ACTA2 and prolyl endopeptidase FAP (Micallef et al., 2012). The CAFs can proliferate, migrate, and secrete ECM factors and abnormal patterns of collagen (Kalluri, 2016). Recently, the heterogeneity of CAFs in different cancer types and their contributions to diverse cancer cell phenotypes have been revealed by several scRNA-seq studies (Li et al., 2017; Puram et al., 2017; Bartoschek et al., 2018; Lambrechts et al., 2018; Ligorio et al., 2019; Dominguez et al., 2020). Accompanying the single-cell landscape of CAFs, novel CAF subtypes with special molecular and functional properties have been identified. For example, Dominguez et al. uncovered a subset of CAFs that are programmed by TGF- $\beta$ and express LRRC15 (Dominguez et al., 2020), which were confirmed to surround tumor islets in patients with pancreatic cancer and correlate with poor response to anti-PD-L1 therapy. Moreover, cross-species single-cell analysis of pancreatic ductal adenocarcinoma identified a new population of antigen-presenting CAFs expressing $\mathrm{MHC}$ class II 
Figure 2. Emerging directions of cancer studies and development of scRNA-seq technologies.

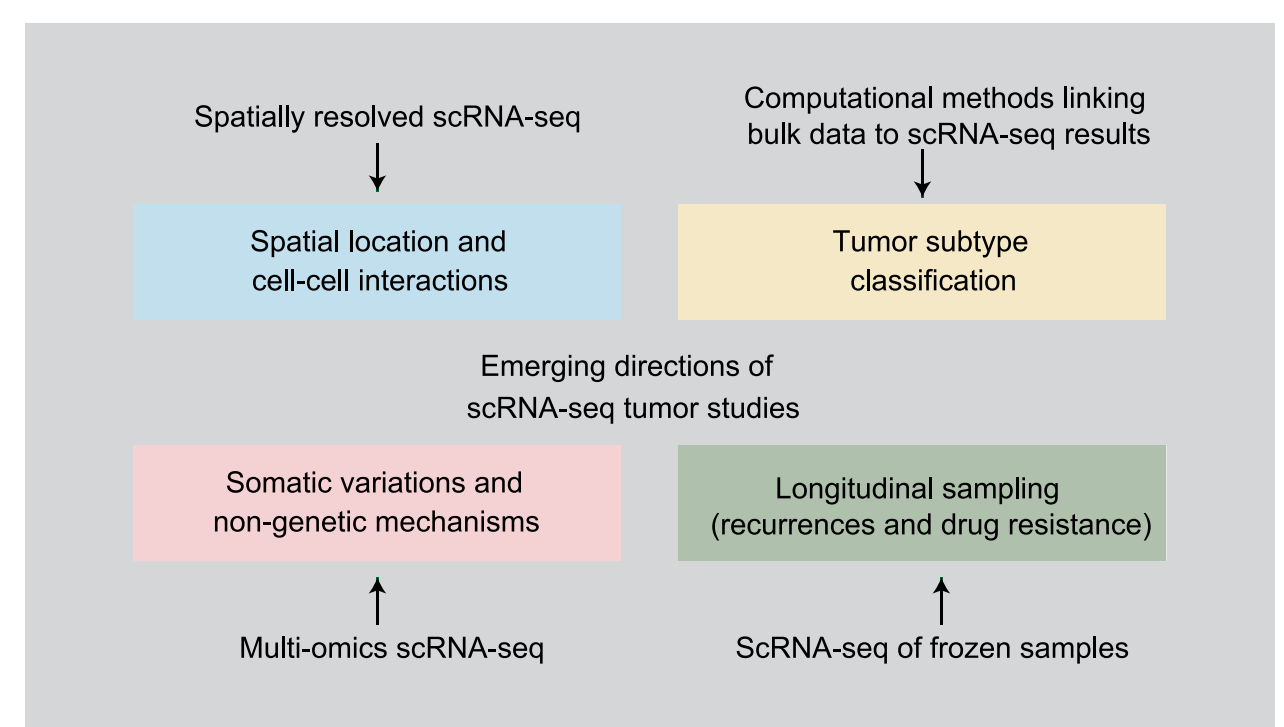

Emerging directions of scRNA-seq tumor studies

Somatic variations and non-genetic mechanisms (recurrences and drug resistance) ScRNA-seq of frozen samples and CD74 but not classic co-stimulatory molecules (Elyada et al., 2019). In an animal model system, antigen-presenting CAFs were shown to have the capability of presenting antigens to $\mathrm{CD}^{+} \mathrm{T}$ cells and modulating the immune response. These new subsets of CAFs offer non-immune targets for combinatorial therapy in the clinical setting. However, the heterogeneity of other stromal cells and their functional features still remain unclear and deserve further exploration at the single-cell level.

\section{CONCLUDING REMARKS}

Cancer is a highly heterogeneous and dynamic disease. Bulk expression profiling of clinical specimens has facilitated our understanding of tumor biology and clinical stratification. However, tumors are increasingly viewed as intricate ecosystems, in which heterogeneous tumor cells interact with diverse immune, endothelial, and stromal cells. The precise characterization of diverse cell types and intra-tumor heterogeneity has been masked by bulk analyses. The rapid development in scRNA-seq technology has laid the foundation for deciphering tumors at single-cell resolution. Here, we review recent studies using ScRNA-seq to address the scientific questions in cancer biology. These studies have revolutionized our understanding of cancer biology by uncovering the cellular landscape of tumors, identifying rare subtypes and their underlying cellular programs, and characterizing the interaction between tumor cells and their microenvironment.

Although great progress has been made in this field, more attention should be paid to the emerging challenges and future directions (Fig. 2). First, the molecular capture efficiency of scRNA-seq technology and bioinformatics approaches should be improved. Secondly, multi-omics technologies require further development to combine mRNA expression analysis with other molecular features such as protein expression levels and DNA methylation. Thirdly, spatially resolved single-cell technology will be a powerful tool to predict cell-cell interactions and provide spatial heterogeneity information. The studies discussed in this review are the inception of single-cell tumor profiling. In the future, further analyses will shed light on cancer biology and lay the foundation for novel therapeutic management.

\section{ABBREVIATIONS}

$\mathrm{AML}$, acute myeloid leukemia; CML, chronic myeloid leukemia; CNV, copy number variation; CSCs, cancer stem cells; ECM, extracellular matrix; ITH, intra-tumor heterogeneity; LSCs, leukemic stem cells; NGS, next-generation sequencing; PDX, patient-derived xenograft; scRNA-seq, single-cell RNA sequencing; TME, tumor microenvironment

\section{FUNDING}

This work was financially supported by the National Science and Technology Major Project (2019YFC1315702) and the Guangdong Province Key Research and Development Program (2019B020226002).

\section{CONFLICTS OF INTEREST/COMPETING INTERESTS}

The authors declare no conflict of interest.

\section{AUTHOR CONTRIBUTIONS}

$\mathrm{YL}, \mathrm{JJ}$, and FB conducted the review and wrote the manuscript. 


\section{OPEN ACCESS}

This article is licensed under a Creative Commons Attribution 4.0 International License, which permits use, sharing, adaptation, distribution and reproduction in any medium or format, as long as you give appropriate credit to the original author(s) and the source, provide a link to the Creative Commons licence, and indicate if changes were made. The images or other third party material in this article are included in the article's Creative Commons licence, unless indicated otherwise in a credit line to the material. If material is not included in the article's Creative Commons licence and your intended use is not permitted by statutory regulation or exceeds the permitted use, you will need to obtain permission directly from the copyright holder. To view a copy of this licence, visit http:// creativecommons.org/licenses/by/4.0/.

\section{REFERENCES}

Affara NI, Ruffell B, Medler TR, Gunderson AJ, Johansson M, Bornstein S, Bergsland E, Steinhoff M, Li Y, Gong Q et al (2014) $B$ cells regulate macrophage phenotype and response to chemotherapy in squamous carcinomas. Cancer Cell 25:809821

Aizarani N, Saviano A, Sagar, Mailly L, Durand S, Herman JS, Pessaux P, Baumert TF, Grün D (2019) A human liver cell atlas reveals heterogeneity and epithelial progenitors. Nature 572:199-204

Amend SR, Roy S, Brown JS, Pienta KJ (2016) Ecological paradigms to understand the dynamics of metastasis. Cancer Lett 380:237-242

Azizi E, Carr AJ, Plitas G, Cornish AE, Konopacki C, Prabhakaran S, Nainys J, Wu K, Kiseliovas V, Setty M et al (2018) Single-cell map of in the breast tumor microenvironment. Cell 174:1293-1308. e1236

Bartoschek M, Oskolkov N, Bocci M, Lovrot J, Larsson C, Sommarin M, Madsen CD, Lindgren D, Pekar G, Karlsson G et al (2018) Spatially and functionally distinct subclasses of breast cancerassociated fibroblasts revealed by single cell RNA sequencing. Nat Commun 9(1):5150

Baryawno N, Przybylski D, Kowalczyk MS, Kfoury Y, Severe N, Gustafsson K, Kokkaliaris KD, Mercier F, Tabaka M, Hofree M et al (2019) A cellular taxonomy of the bone marrow stroma in homeostasis and leukemia. Cell 177:1915-1932.e1916

Baslan T, Hicks J (2017) Unravelling biology and shifting paradigms in cancer with single-cell sequencing. Nat Rev Cancer 17:557569

Baslan T, Kendall J, Rodgers L, Cox H, Riggs M, Stepansky A, Troge J, Ravi K, Esposito D, Lakshmi B et al (2012) Genomewide copy number analysis of single cells. Nat Protoc 7:10241041

Berger MF, Mardis ER (2018) The emerging clinical relevance of genomics in cancer medicine. Nat Rev Clin Oncol 15:353-365

Bi K, He MX, Bakouny Z, Kanodia A, Napolitano S, Wu J, Grimaldi G, Braun DA, Cuoco MS, Mayorga A et al (2021) Tumor and immune reprogramming during immunotherapy in advanced renal cell carcinoma. Cancer Cell 39:649-661.e645
Binnewies M, Roberts EW, Kersten K, Chan V, Fearon DF, Merad M, Coussens LM, Gabrilovich DI, Ostrand-Rosenberg S, Hedrick CC et al (2018) Understanding the tumor immune microenvironment (TIME) for effective therapy. Nat Med 24:541-550

Brodbeck T, Nehmann N, Bethge A, Wedemann G, Schumacher U (2014) Perforin-dependent direct cytotoxicity in natural killer cells induces considerable knockdown of spontaneous lung metastases and computer modelling-proven tumor cell dormancy in a HT29 human colon cancer xenograft mouse model. Mol Cancer 13:244

Burrell RA, McGranahan N, Bartek J, Swanton C (2013) The causes and consequences of genetic heterogeneity in cancer evolution. Nature 501:338-345

Cancer Genome Atlas Research Network (2012a) Comprehensive genomic characterization of squamous cell lung cancers. Nature 489:519-525

Cancer Genome Atlas Research Network (2012b) Comprehensive genomic characterization of squamous cell lung cancers The Cancer Genome Atlas Research Network (vol 489, pg 519, 2012). Nature 491:288-288

Cancer Genome Atlas Research Network (2014) Comprehensive molecular profiling of lung adenocarcinoma. Nature 511:543-550

Cancer Genome Atlas Research Network, Linehan WM, Spellman PT, Ricketts CJ, Creighton CJ, Fei SS, Davis C, Wheeler DA, Murray BA, Schmidt L et al (2016) Comprehensive molecular characterization of papillary renal-cell carcinoma. N Engl J Med 374:135-145

Chen DS, Mellman I (2017) Elements of cancer immunity and the cancer-immune set point. Nature 541:321-330

Chen YC, Sahoo S, Brien R, Jung S, Humphries B, Lee W, Cheng YH, Zhang Z, Luker KE, Wicha MS et al (2019) Single-cell RNAsequencing of migratory breast cancer cells: discovering genes associated with cancer metastasis. Analyst 144:7296-7309

Chung W, Eum HH, Lee HO, Lee KM, Lee HB, Kim KT, Ryu HS, Kim S, Lee JE, Park YH et al (2017) Single-cell RNA-seq enables comprehensive tumour and immune cell profiling in primary breast cancer. Nat Commun 8:15081

Cillo AR, Kurten CHL, Tabib T, Qi Z, Onkar S, Wang T, Liu A, Duvvuri U, Kim S, Soose RJ et al (2020) Immune landscape of viral- and carcinogen-driven head and neck cancer. Immunity 52:183-199. e189

Clevers $H$ (2011) The cancer stem cell: premises, promises and challenges. Nat Med 17:313-319

de Groot AE, Roy S, Brown JS, Pienta KJ, Amend SR (2017) Revisiting seed and soil: examining the primary tumor and cancer cell foraging in metastasis. Mol Cancer Res 15:361-370

Dean M, Fojo T, Bates S (2005) Tumour stem cells and drug resistance. Nat Rev Cancer 5:275-284

Dominguez CX, Muller S, Keerthivasan S, Koeppen H, Hung J, Gierke S, Breart B, Foreman O, Bainbridge TW, Castiglioni A et al (2020) Single-cell RNA sequencing reveals stromal evolution into LRRC15(+) myofibroblasts as a determinant of patient response to cancer immunotherapy. Cancer Discov 10:232-253

Elyada E, Bolisetty M, Laise P, Flynn WF, Courtois ET, Burkhart RA, Teinor JA, Belleau P, Biffi G, Lucito MS et al (2019) Cross-species single-cell analysis of pancreatic ductal adenocarcinoma reveals 
antigen-presenting cancer-associated fibroblasts. Cancer Discov 9:1102-1123

Engblom C, Pfirschke C, Pittet MJ (2016) The role of myeloid cells in cancer therapies. Nat Rev Cancer 16:447-462

Fearon ER, Vogelstein B (1990) A genetic model for colorectal tumorigenesis. Cell 61:759-767

Filbin MG, Tirosh I, Hovestadt V, Shaw ML, Escalante LE, Mathewson ND, Neftel C, Frank N, Pelton K, Hebert CM et al (2018) Developmental and oncogenic programs in H3K27M gliomas dissected by single-cell RNA-seq. Science 360:331-335

Garraway LA, Lander ES (2013) Lessons from the cancer genome. Cell 153:17-37

George J, Lim JS, Jang SJ, Cun Y, Ozretic L, Kong G, Leenders F, Lu X, Fernandez-Cuesta L, Bosco G et al (2015) Comprehensive genomic profiles of small cell lung cancer. Nature 524:47-53

Gerlinger M, Rowan AJ, Horswell S, Math M, Larkin J, Endesfelder D, Gronroos E, Martinez P, Matthews N, Stewart A et al (2012) Intratumor heterogeneity and branched evolution revealed by multiregion sequencing. N Engl J Med 366:883-892

Giustacchini A, Thongjuea S, Barkas N, Woll PS, Povinelli BJ, Booth CAG, Sopp P, Norfo R, Rodriguez-Meira A, Ashley N et al (2017) Single-cell transcriptomics uncovers distinct molecular signatures of stem cells in chronic myeloid leukemia. Nat Med 23:692-702

Guo W, Li L, He J, Liu Z, Han M, Li F, Xia X, Zhang X, Zhu Y, Wei Y et al (2020) Single-cell transcriptomics identifies a distinct luminal progenitor cell type in distal prostate invagination tips. Nat Genet 52:908-918

Guo X, Zhang Y, Zheng L, Zheng C, Song J, Zhang Q, Kang B, Liu $Z$, Jin L, Xing R et al (2018) Global characterization of T cells in non-small-cell lung cancer by single-cell sequencing. Nat Med 24:978-985

Hashimshony T, Senderovich N, Avital G, Klochendler A, de Leeuw Y, Anavy L, Gennert D, Li SQ, Livak KJ, Rozenblatt-Rosen O et al (2016) CEL-Seq2: sensitive highly-multiplexed single-cell RNASeq. Genome Biol 17:77

Hermann PC, Huber SL, Herrler T, Aicher A, Ellwart JW, Guba M, Bruns CJ, Heeschen C (2007) Distinct populations of cancer stem cells determine tumor growth and metastatic activity in human pancreatic cancer. Cell Stem Cell 1:313-323

Hollern DP, Xu N, Thennavan A, Glodowski C, Garcia-Recio S, Mott KR, He X, Garay JP, Carey-Ewend K, Marron D et al (2019) B cells and $T$ follicular helper cells mediate response to checkpoint inhibitors in high mutation burden mouse models of breast cancer. Cell 179:1191-1206.e1121

Horning AM, Wang Y, Lin CK, Louie AD, Jadhav RR, Hung CN, Wang CM, Lin CL, Kirma NB, Liss MA et al (2018) Single-cell RNA-seq reveals a subpopulation of prostate cancer cells with enhanced cell-cycle-related transcription and attenuated androgen response. Cancer Res 78:853-864

Jan M, Snyder TM, Corces-Zimmerman MR, Vyas P, Weissman IL, Quake SR, Majeti R (2012) Clonal evolution of preleukemic hematopoietic stem cells precedes human acute myeloid leukemia. Sci Transl Med. https://doi.org/10.1126/scitransImed. 3004315

Jerby-Arnon L, Shah P, Cuoco MS, Rodman C, Su MJ, Melms JC, Leeson R, Kanodia A, Mei S, Lin JR et al (2018) A cancer cell program promotes T cell exclusion and resistance to checkpoint blockade. Cell 175:984-997.e924

Jin S, Li R, Chen MY, Yu C, Tang LQ, Liu YM, Li JP, Liu YN, Luo YL, Zhao $Y$ et al (2020) Single-cell transcriptomic analysis defines the interplay between tumor cells, viral infection, and the microenvironment in nasopharyngeal carcinoma. Cell Res 30:950-965

Jordan NV, Bardia A, Wittner BS, Benes C, Ligorio M, Zheng Y, Yu $M$, Sundaresan TK, Licausi JA, Desai $R$ et al (2016) HER2 expression identifies dynamic functional states within circulating breast cancer cells. Nature 537:102-106

Just NP, Finlay-Schultz J, Gillen AE, Cittelly DM, Wellberg EA, Horwitz KB, Kabos P, Sartorius CA (2018) Single-cell RNA sequencing defines regulatory networks in ER plus breast cancer organ-specific metastases. Cancer Res. https://doi.org/10.1158/ 1538-7445.AM2018-3027

Kalluri R (2016) The biology and function of fibroblasts in cancer. Nat Rev Cancer 16:582-598

Kim KT, Lee HW, Lee HO, Song HJ, Jeong DE, Shin S, Kim H, Shin Y, Nam DH, Jeong BC et al (2016) Application of single-cell RNA sequencing in optimizing a combinatorial therapeutic strategy in metastatic renal cell carcinoma. Genome Biol 17:80

Klein-Goldberg A, Maman S, Witz IP (2014) The role played by the microenvironment in site-specific metastasis. Cancer Lett 352:54-58

Klein AM, Mazutis L, Akartuna I, Tallapragada N, Veres A, Li V, Peshkin L, Weitz DA, Kirschner MW (2015) Droplet barcoding for single-cell transcriptomics applied to embryonic stem cells. Cell 161:1187-1201

Kreso A, Dick JE (2014) Evolution of the cancer stem cell model. Cell Stem Cell 14:275-291

Kurtulus S, Madi A, Escobar G, Klapholz M, Nyman J, Christian E, Pawlak M, Dionne D, Xia JR, Rozenblatt-Rosen O et al (2019) Checkpoint blockade immunotherapy induces dynamic changes in PD- $1^{(-)} \mathrm{CD}^{(+)}$tumor-infiltrating T cells. Immunity 50:181

Lambert AW, Pattabiraman DR, Weinberg RA (2017) Emerging biological principles of metastasis. Cell 168:670-691

Lambrechts D, Wauters E, Boeckx B, Aibar S, Nittner D, Burton O, Bassez A, Decaluwe H, Pircher A, Van den Eynde K et al (2018) Phenotype molding of stromal cells in the lung tumor microenvironment. Nat Med 24:1277

Lavin Y, Kobayashi S, Leader A, Amir ED, Elefant N, Bigenwald C, Remark R, Sweeney R, Becker CD, Levine JH et al (2017) Innate immune landscape in early lung adenocarcinoma by paired single-cell analyses. Cell 169:750-765.e717

Lawson DA, Bhakta NR, Kessenbrock K, Prummel KD, Yu Y, Takai K, Zhou A, Eyob H, Balakrishnan S, Wang CY et al (2015) Singlecell analysis reveals a stem-cell program in human metastatic breast cancer cells. Nature 526:131-135

Leruste A, Tosello J, Ramos RN, Tauziede-Espariat A, Brohard S, Han ZY, Beccaria K, Andrianteranagna M, Caudana P, Nikolic J et al (2019) Clonally expanded T cells reveal immunogenicity of rhabdoid tumors. Cancer Cell 36:597

Li H, Courtois ET, Sengupta D, Tan Y, Chen KH, Goh JJL, Kong SL, Chua C, Hon LK, Tan WS et al (2017) Reference component analysis of single-cell transcriptomes elucidates cellular heterogeneity in human colorectal tumors. Nat Genet 49:708-718 
Li HJ, van der Leun AM, Yofe I, Lubling Y, Gelbard-Solodkin D, van Akkooi ACJ, van den Braber M, Rozeman EA, Haanen JBAG, Blank CU et al (2019) Dysfunctional CD8 T cells form a proliferative, dynamically regulated compartment within human melanoma. Cell 176:775

Ligorio M, Sil S, Malagon-Lopez J, Nieman LT, Misale S, Di Pilato M, Ebright RY, Karabacak MN, Kulkarni AS, Liu A et al (2019) Stromal microenvironment shapes the intratumoral architecture of pancreatic cancer. Cell 178:160

Lu Y, Zhao Q, Liao JY, Song E, Xia Q, Pan J, Li Y, Li J, Zhou B, Ye Y et al (2020) Complement signals determine opposite effects of $B$ cells in chemotherapy-induced immunity. Cell 180(6):1081-1097

Maley CC, Aktipis A, Graham TA, Sottoriva A, Boddy AM, Janiszewska M, Silva AS, Gerlinger M, Yuan YY, Pienta KJ et al (2017) Classifying the evolutionary and ecological features of neoplasms. Nat Rev Cancer 17:605-619

Maman S, Witz IP (2018) A history of exploring cancer in context. Nat Rev Cancer 18:359-376

McGranahan N, Swanton C (2017) Clonal heterogeneity and tumor evolution: past, present, and the future. Cell 168:613-628

Merlo LMF, Pepper JW, Reid BJ, Maley CC (2006) Cancer as an evolutionary and ecological process. Nat Rev Cancer 6:924-935

Miao Y, Yang H, Levorse J, Yuan S, Polak L, Sribour M, Singh B, Rosenblum MD, Fuchs E (2019) Adaptive immune resistance emerges from tumor-initiating stem cells. Cell 177:1172-1186. e1114

Micallef L, Vedrenne N, Billet F, Coulomb B, Darby IA, Desmouliere A (2012) The myofibroblast, multiple origins for major roles in normal and pathological tissue repair. Fibrogenesis Tissue Repair 5:S5

Miyamoto DT, Zheng Y, Wittner BS, Lee RJ, Zhu H, Broderick KT, Desai R, Fox DB, Brannigan BW, Trautwein J et al (2015) RNASeq of single prostate CTCs implicates noncanonical Wnt signaling in antiandrogen resistance. Science 349:1351-1356

Muller S, Kohanbash G, Liu SJ, Alvarado B, Carrera D, Bhaduri A, Watchmaker PB, Yagnik G, Di Lullo E, Malatesta M et al (2017) Single-cell profiling of human gliomas reveals macrophage ontogeny as a basis for regional differences in macrophage activation in the tumor microenvironment. Genome Biol 18:234

Murugaesu N, Wilson GA, Birkbak NJ, Watkins T, McGranahan N, Kumar S, Abbassi-Ghadi N, Salm M, Mitter R, Horswell S et al (2015) Tracking the genomic evolution of esophageal adenocarcinoma through neoadjuvant chemotherapy. Cancer Discov 5:821-831

Nakagawa H, Fujita M (2018) Whole genome sequencing analysis for cancer genomics and precision medicine. Cancer Sci 109:513-522

Navin N, Kendall J, Troge J, Andrews P, Rodgers L, Mclndoo J, Cook K, Stepansky A, Levy D, Esposito D et al (2011) Tumour evolution inferred by single-cell sequencing. Nature 472:90-94

Neftel C, Laffy J, Filbin MG, Hara T, Shore ME, Rahme GJ, Richman AR, Silverbush D, Shaw ML, Hebert CM et al (2019) An integrative model of cellular states, plasticity, and genetics for glioblastoma. Cell 178:835-849.e821

Orimo A, Weinberg RA (2006) Stromal fibroblasts in cancer-a novel tumor-promoting cell type. Cell Cycle 5:1597-1601
Pantel K, Brakenhoff RH (2004) Dissecting the metastatic cascade. Nat Rev Cancer 4:448-456

Pastushenko I, Brisebarre A, Sifrim A, Fioramonti M, Revenco T, Boumahdi S, Van Keymeulen A, Brown D, Moers V, Lemaire S et al (2018) Identification of the tumour transition states occurring during EMT. Nature 556:463-468

Patel AP, Tirosh I, Trombetta JJ, Shalek AK, Gillespie SM, Wakimoto H, Cahill DP, Nahed BV, Curry WT, Martuza RL et al (2014) Single-cell RNA-seq highlights intratumoral heterogeneity in primary glioblastoma. Science 344:1396-1401

Paulson KG, Voillet V, McAfee MS, Hunter DS, Wagener FD, Perdicchio M, Valente WJ, Koelle SJ, Church CD, Vandeven N et al (2018) Acquired cancer resistance to combination immunotherapy from transcriptional loss of class I HLA. Nat Commun 9:3868

Picelli S, Bjorklund AK, Faridani OR, Sagasser S, Winberg G, Sandberg R (2013) Smart-seq2 for sensitive full-length transcriptome profiling in single cells. Nat Methods 10:1096-1098

Puram SV, Tirosh I, Parikh AS, Patel AP, Yizhak K, Gillespie S, Rodman C, Luo CL, Mroz EA, Emerick KS et al (2017) Single-cell transcriptomic analysis of primary and metastatic tumor ecosystems in head and neck cancer. Cell 171:1611-1624.e1624

Pylayeva-Gupta Y, Das S, Handler JS, Hajdu CH, Coffre M, Koralov SB, Bar-Sagi D (2016) IL35-producing B cells promote the development of pancreatic neoplasia. Cancer Discov 6:247-255

Rambow F, Rogiers A, Marin-Bejar O, Aibar S, Femel J, Dewaele M, Karras P, Brown D, Chang YH, Debiec-Rychter M et al (2018) Toward minimal residual disease-directed therapy in melanoma. Cell 174:843

Ramskold D, Luo SJ, Wang YC, Li R, Deng QL, Faridani OR, Daniels GA, Khrebtukova I, Loring JF, Laurent LC et al (2012) Full-length mRNA-Seq from single-cell levels of RNA and individual circulating tumor cells. Nat Biotechnol 30:777-782

Ren X, Kang B, Zhang Z (2018) Understanding tumor ecosystems by single-cell sequencing: promises and limitations. Genome Biol 19:211

Salmon H, Idoyaga J, Rahman A, Leboeuf M, Remark R, Jordan S, Casanova-Acebes M, Khudoynazarova M, Agudo J, Tung $\mathrm{N}$ et al (2016) Expansion and activation of $\mathrm{CD} 103^{(+)}$dendritic cell progenitors at the tumor site enhances tumor responses to therapeutic PD-L1 and BRAF inhibition. Immunity 44:924-938

Savage P, Blanchet-Cohen A, Revil T, Badescu D, Saleh SMI, Wang YC, Zuo D, Liu L, Bertos NR, Munoz-Ramos V et al (2017) A targetable EGFR-dependent tumor-initiating program in breast cancer. Cell Rep 21:1140-1149

Savas P, Virassamy B, Ye C, Salim A, Mintoff CP, Caramia F, Salgado R, Byrne DJ, Teo ZL, Dushyanthen S et al (2018) Single-cell profiling of breast cancer $T$ cells reveals a tissue-resident memory subset associated with improved prognosis. Nat Med 24:986-993

Sceneay J, Chow MT, Chen A, Halse HM, Wong CSF, Andrews DM, Sloan EK, Parker BS, Bowtell DD, Smyth MJ et al (2012) Primary tumor hypoxia recruits $\mathrm{CD} 11 \mathrm{~b}(+) / \mathrm{Ly} 6 \mathrm{C}(\mathrm{med}) / \mathrm{Ly} 6 \mathrm{G}(+)$ immune suppressor cells and compromises NK cell cytotoxicity in the premetastatic niche. Cancer Res 72:3906-3911

Singer M, Wang C, Cong L, Marjanovic ND, Kowalczyk MS, Zhang H, Nyman J, Sakuishi K, Kurtulus S, Gennert D et al (2016) A 
distinct gene module for dysfunction uncoupled from activation in tumor-infiltrating T cells. Cell 166:1500-1511.e1509

Song QQ, Hawkins GA, Wudel L, Chou PC, Forbes E, Pullikuth AK, Liu L, Jin GX, Craddock L, Topaloglu U et al (2019) Dissecting intratumoral myeloid cell plasticity by single cell RNA-seq. Cancer Med US 8:3072-3085

Sottoriva A, Kang H, Ma Z, Graham TA, Salomon MP, Zhao J, Marjoram P, Siegmund K, Press MF, Shibata D et al (2015) A Big Bang model of human colorectal tumor growth. Nat Genet 47:209-216

Suva ML, Tirosh I (2019) Single-cell RNA sequencing in cancer: lessons learned and emerging challenges. Mol Cell 75:7-12

Swanton C, Govindan R (2016) Clinical implications of genomic discoveries in lung cancer. N Engl J Med 374:1864-1873

Szczerba BM, Castro-Giner F, Vetter M, Krol I, Gkountela S, Landin J, Scheidmann MC, Donato C, Scherrer R, Singer J et al (2019) Neutrophils escort circulating tumour cells to enable cell cycle progression. Nature 566:553

Tang FC, Barbacioru C, Wang YZ, Nordman E, Lee C, Xu NL, Wang $\mathrm{XH}$, Bodeau J, Tuch BB, Siddiqui A et al (2009) mRNA-Seq whole-transcriptome analysis of a single cell. Nat Methods 6:377-386

Tirosh I, Izar B, Prakadan SM, Wadsworth MH II, Treacy D, Trombetta JJ, Rotem A, Rodman C, Lian C, Murphy G et al (2016a) Dissecting the multicellular ecosystem of metastatic melanoma by single-cell RNA-seq. Science 352:189-196

Tirosh I, Suva ML (2019) Deciphering human tumor biology by single-cell expression profiling. Annu Rev Cancer Biol 3:151-166

Tirosh I, Venteicher AS, Hebert C, Escalante LE, Patel AP, Yizhak K, Fisher JM, Rodman C, Mount C, Filbin MG et al (2016b) Singlecell RNA-seq supports a developmental hierarchy in human oligodendroglioma. Nature 539:309-313

Turajlic S, Sottoriva A, Graham T, Swanton C (2019) Resolving genetic heterogeneity in cancer. Nat Rev Genet 20:404-416

Turajlic S, Swanton C (2017) Implications of cancer evolution for drug development. Nat Rev Drug Discov 16:441-442

Valkenburg KC, de Groot AE, Pienta KJ (2018) Targeting the tumour stroma to improve cancer therapy. Nat Rev Clin Oncol 15:366381

van Galen P, Hovestadt V, Wadsworth li MH, Hughes TK, Griffin GK, Battaglia S, Verga JA, Stephansky J, Pastika TJ, Lombardi Story $J$ et al (2019) Single-cell RNA-Seq reveals AML hierarchies relevant to disease progression and immunity. Cell 176:12651281.e1224

Velten L, Story BA, Hernandez-Malmierca P, Raffel S, Leonce DR, Milbank J, Paulsen M, Demir A, Szu-Tu C, Fromel R et al (2021) Identification of leukemic and pre-leukemic stem cells by clonal tracking from single-cell transcriptomics. Nat Commun 12:1366

Venteicher AS, Tirosh I, Hebert C, Yizhak K, Neftel C, Filbin MG, Hovestadt V, Escalante LE, Shaw ML, Rodman C et al (2017) Decoupling genetics, lineages, and microenvironment in IDHmutant gliomas by single-cell RNA-seq. Science. https://doi.org/ 10.1126/science.aai8478

Vermeulen L, Snippert HJ (2014) Stem cell dynamics in homeostasis and cancer of the intestine. Nat Rev Cancer 14:468-480

Vladoiu MC, El-Hamamy I, Donovan LK, Farooq H, Holgado BL, Sundaravadanam Y, Ramaswamy V, Hendrikse LD, Kumar S,
Mack SC et al (2019) Childhood cerebellar tumours mirror conserved fetal transcriptional programs. Nature 572:67-73

Wan LL, Pantel K, Kang YB (2013) Tumor metastasis: moving new biological insights into the clinic. Nat Med 19:1450-1464

Wang QH, Hu X, Muller F, Kim H, Squatrito M, Mikkelsen T, Scarpace L, Barthel F, Lin YH, Satani N et al (2016) Tumor evolution of glioma intrinsic gene expression subtype associates with immunological changes in the microenvironment. NeuroOncology 18:202-202

Weinberg RA (2002) Cancer Biology and Therapy: the road ahead. Cancer Biol Ther 1:3

Weis SM, Cheresh DA (2011) Tumor angiogenesis: molecular pathways and therapeutic targets. Nat Med 17:1359-1370

Williams MJ, Werner B, Barnes CP, Graham TA, Sottoriva A (2016) Identification of neutral tumor evolution across cancer types. Nat Genet 48:238-244

Xing X, Yang F, Huang Q, Guo H, Li J, Qu M, Bai F, Wang J (2021) Decoding the multicellular ecosystem of lung adenocarcinoma manifested as pulmonary subsolid nodules by single-cell RNA sequencing. Sci Adv. https://doi.org/10.1126/sciadv.abd9738

Yamamoto R, Morita Y, Ooehara J, Hamanaka S, Onodera M, Rudolph KL, Ema H, Nakauchi H (2013) Clonal analysis unveils self-renewing lineage-restricted progenitors generated directly from hematopoietic stem cells. Cell 154:1112-1126

Yost KE, Satpathy AT, Wells DK, Qi YY, Wang CL, Kageyama R, McNamara KL, Granja JM, Sarin KY, Brown RA et al (2019) Clonal replacement of tumor-specific $T$ cells following PD-1 blockade. Nat Med 25:1251

Young MD, Mitchell TJ, Braga FAV, Tran MGB, Stewart BJ, Ferdinand JR, Collord G, Botting RA, Popescu DM, Loudon $\mathrm{KW}$ et al (2018) Single-cell transcriptomes from human kidneys reveal the cellular identity of renal tumors. Science 361:594

Zhang L, Yu X, Zheng L, Zhang Y, Li Y, Fang Q, Gao R, Kang B, Zhang Q, Huang JY et al (2018) Lineage tracking reveals dynamic relationships of $\mathrm{T}$ cells in colorectal cancer. Nature 564:268-272

Zhang L, Zhang Z (2019) Recharacterizing tumor-infiltrating lymphocytes by single-cell RNA sequencing. Cancer Immunol Res 7:1040-1046

Zhang Q, He Y, Luo N, Patel SJ, Han Y, Gao R, Modak M, Carotta S, Haslinger C, Kind D et al (2019) Landscape and dynamics of single immune cells in hepatocellular carcinoma. Cell 179:829$845 . e 820$

Zheng C, Zheng L, Yoo JK, Guo H, Zhang Y, Guo X, Kang B, Hu R, Huang JY, Zhang Q et al (2017a) Landscape of infiltrating T cells in liver cancer revealed by single-cell sequencing. Cell 169:13421356.e1316

Zheng GX, Terry JM, Belgrader P, Ryvkin P, Bent ZW, Wilson R, Ziraldo SB, Wheeler TD, McDermott GP, Zhu J et al (2017b) Massively parallel digital transcriptional profiling of single cells. Nat Commun 8:14049

Zheng H, Pomyen Y, Hernandez MO, Li C, Livak F, Tang W, Dang H, Greten TF, Davis JL, Zhao $Y$ et al (2018) Single-cell analysis reveals cancer stem cell heterogeneity in hepatocellular carcinoma. Hepatology 68:127-140 